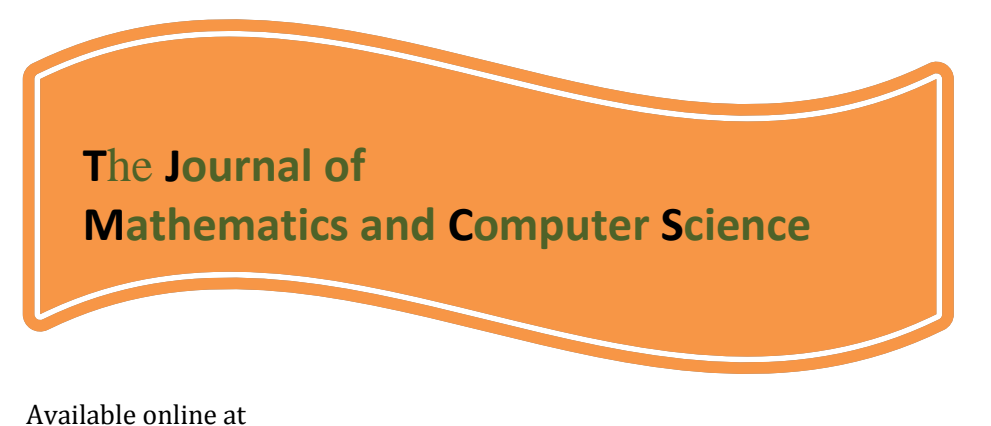

\title{
http://www.TIMCS.com
}

The Journal of Mathematics and Computer Science Vol .5 No.4 (2012) 258-264

\section{Solution of Fredholm Integro-Differential Equations System by Modified Decomposition Method}

\author{
M. Rabbani \\ Department of Mathematics, Sari Branch, Islamic Azad University, Sari, Iran \\ mrabbani@iausari.ac.ir \\ B. Zarali ${ }^{1}$ \\ Department of Mathematics, Science and Research Branch, Islamic Azad University, \\ Mazandaran, Iran \\ behrozzarali@yahoo.com
}

Received: February 2012, Revised: November 2012

Online Publication: December 2012

\begin{abstract}
In this paper, the technique of modified decomposition method is used to solve a system of linear integro-differential equations with initial conditions. Moreover, two particular examples are discussed to show relability and the performance of the modified decomposition method.
\end{abstract}

Keywords: Modified decomposition method; System of Fredholm integro-differential equations.

\section{INTRODUCTION}

Some important problems in science and engineering can usually be reduced to a system of integral and integro-differential equations. Integro-differential equation has attracted much attention and solving this equation has been one of the interesting tasks for mathematicians. In this research we try to introduce a solution of system of linear integro-differential equations the following form:

${ }^{1}$ Corresponding author 


$$
\left\{\begin{array}{c}
u_{1}^{\left(n_{1}\right)}(x)=f_{1}(x)+\int_{\alpha_{1}}^{\beta_{1}} k_{1}\left(x, t, u_{1}(t), u_{2}(t), \ldots, u_{p}(t)\right) d t \\
u_{2}^{\left(n_{2}\right)}(x)=f_{2}(x)+\int_{\alpha_{2}}^{\beta_{2}} k_{2}\left(x, t, u_{1}(t), u_{2}(t), \ldots, u_{p}(t)\right) d t \\
\vdots \\
u_{p}^{\left(n_{p}\right)}(x)=f_{p}(x)+\int_{\alpha_{p}}^{\beta_{p}} k_{p}\left(x, t, u_{1}(t), u_{2}(t), \ldots, u_{p}(t)\right) d t
\end{array}\right.
$$

With initial conditions:

$u_{i}^{(j)}\left(x_{0}\right)=u_{i j}, i=1, \ldots, p, j=0,1, \ldots, n_{i}-1$.

Many researchers are shown in solving such types of integro-differential equations system. Recently, Biazar in [4], used the Adomian decomposition method for solving the system of integro-differentioal equations also Davari applied the some method for solving linear Fredholm integro-differential equations. Maleknejad in [9], used the Galerkin methods with Hybrid Functions for Solving linear integro-differential equation system. Biazar et al. in[5] by He's Homotopy perturbation method for systems of integro-differential equations, Arikoglu and Ozkol in[2], by using differential Transform method, Maleknejad et al. in [8] by using rationalized Haar functions method can be solved to integro-differential equations system. Pour-Mahmoud et al. in [10] presented the Tau method for the numerical solution of systems of Fredholm integro-differential equations.

In this article, we introduce a modified decomposition method for solving Eq.(1).The modified technique of Adomian decomposition method developed by Wazwaz in [11, 13] will form a useful basis for studying the system of Fredholm integro-differentioal equations. The modified decomposition method, in [11, 13], has a constructive attraction in that it provides the exact solution by computing only two terms of the decomposition series.

\section{MODIFIED DECOMPOSITION METHOD}

Consider a general functional equation

$L u+R u+N u=f(x)$,

Where $u(x)$ is the unknown function and the linear terms are decomposed into $L+R$ and $N u$ indicates the nonlinear terms. Since $L$ is easily invertible and $R$ is the remainder of the nonlinear operator and $f(x)$ is the source term. From Eq. (2)

$L u=f(x)-R u-N u$,

applying $L^{-1}$ on the both sides of above equation andusing the given conditions, we get

$u(x)=g(x)-L^{-1}(R u)-L^{-1}(N u)$

where $L^{-1}(f(x))=g(x)$.

Employing the Adomian method the series solution $u(x)$ is defined as:

$u(x)=\sum_{n=0}^{\infty} u_{n}(x)$, 
the components $u_{0}, u_{1}, u_{2}, \ldots$ can easily be determinerecursively from the following relations:

$u_{0}(x)=g(x)$

$$
u_{n+1}(x)=-L^{-1}\left(R u_{n}\right)-L^{-1}\left(N u_{n}\right), \quad n \geq 0 .
$$

The decomposition method usually identifies the zero thcomponents $u_{0}(x)$ as the function $g(x)$. But modified decomposition method suggest that the function $g(x)$ defined above in Eq. (4) can bedecomposed into two parts namely, $g_{0}(x)$ and $g_{1}(x)$,i.e,

$g(x)=g_{0}(x)+g_{1}(x)$.

In the above equation proper choice of $g_{0}(x)$ and $g_{1}(x)$ is essential and depends mainly on the trail basis.Thus the following recursive relations for the modified decomposition method are formulated as:

$u_{0}(x)=g_{0}(x)$

$u_{1}(x)=g_{1}(x)-L^{-1}\left(R u_{0}\right)-L^{-1}\left(N u_{0}\right)$,

$u_{n+1}(x)=-L^{-1}\left(R u_{n}\right)-L^{-1}\left(N u_{n}\right), \quad n \geq 1$.

Eq. (8) demonstrates reliability in that, it accelerates the convergence of the solution and reduces the series of computation as compared to Adomian decomposition method.

\section{SOLUTION OF THE PROBLEM}

We suppose $L$ be the operator $L=\frac{d}{d x}$ and $L^{-1}$ its inverse operator.Now if act $n_{i}$ times operator $L^{-1}$ on each equation of the system (1) and if we define $\underbrace{L^{-1} \ldots L^{-1}}_{n_{i} \text { times }}=c_{i}, \mathrm{i}=1,2, \ldots, \mathrm{p}$. we get

$\left\{\begin{array}{c}u_{1}(x)=g_{1}(x)+\int_{\alpha_{1}}^{\beta_{1}} c_{1} k_{1}\left(x, t, u_{1}(t), u_{2}(t), \ldots, u_{p}(t)\right) d t \\ u_{2}(x)=g_{2}(x)+\int_{\alpha_{2}}^{\beta_{2}} c_{2} k_{2}\left(x, t, u_{1}(t), u_{2}(t), \ldots, u_{p}(t)\right) d t \\ \vdots \\ u_{p}(x)=g_{p}(x)+\int_{\alpha_{p}}^{\beta_{p}} c_{p} k_{p}\left(x, t, u_{1}(t), u_{2}(t), \ldots, u_{p}(t)\right) d t\end{array}\right.$

in which

$\left\{\begin{array}{c}g_{1}(x)=c_{1} f_{1}(x) \\ g_{2}(x)=c_{2} f_{2}(x) \\ \vdots \\ g_{p}(x)=c_{p} f_{p}(x)\end{array}\right.$

Or briefly $g_{i}(x)=c_{i} f_{i}(x), i=1,2, \ldots$.

Adomian decomposition method decomposed the solution of $u_{i}(\mathrm{x})$ as the following:

$\left\{\begin{array}{c}u_{1}(x)=\sum_{j=0}^{\infty} u_{1 j}(x), \\ u_{2}(x)=\sum_{j=0}^{\infty} u_{2 j}(x), \\ \vdots \\ u_{p}(x)=\sum_{j=0}^{\infty} u_{p j}(x) .\end{array}\right.$ 
Or briefly $u_{i}(x)=\sum_{j=0}^{\infty} u_{i j}(x), \quad i=1,2, \ldots$.

By substituting (10) in (9), we get

$$
\left\{\begin{array}{c}
\sum_{j=0}^{\infty} u_{1 j}(x)=g_{1}(x)+\int_{\alpha_{1}}^{\beta_{1}} c_{1} k_{1}\left(x, t, \sum_{j=0}^{\infty} u_{1 j}(t), \ldots, \sum_{j=0}^{\infty} u_{p j}(t)\right) d t, \\
\sum_{j=0}^{\infty} u_{2 j}(x)=g_{2}(x)+\int_{\alpha_{2}}^{\beta_{2}} c_{2} k_{2}\left(x, t, \sum_{j=0}^{\infty} u_{1 j}(t), \ldots, \sum_{j=0}^{\infty} u_{p j}(t)\right) d t \\
\vdots \\
\sum_{j=0}^{\infty} u_{p j}(x)=g_{p}(x)+\int_{\alpha_{p}}^{\beta_{p}} c_{p} k_{p}\left(x, t, \sum_{j=0}^{\infty} u_{1 j}(t), \ldots, \sum_{j=0}^{\infty} u_{p j}(t)\right) d t .
\end{array}\right.
$$

By using modified decomposition method we decompose function $g_{i}(x)$ as follows:

$g_{i}(x)=g_{i 0}(x)+g_{i 1}(x)$.

We set

$$
\left\{\begin{array}{c}
u_{10}(x)=g_{10}(x) \\
u_{20}(x)=g_{20}(x) \\
\vdots \\
u_{p 0}(x)=g_{p 0}(x) .
\end{array}\right.
$$

And

$$
\left\{\begin{array}{c}
u_{11}(x)=g_{11}(x)+\int_{\alpha_{1}}^{\beta_{1}} c_{1} k_{1}\left(x, t, u_{10}(t), u_{20}(t), \ldots, u_{p 0}(t)\right) d t \\
u_{21}(x)=g_{21}(x)+\int_{\alpha_{2}}^{\beta_{2}} c_{2} k_{2}\left(x, t, u_{10}(t), u_{20}(t), \ldots, u_{p 0}(t)\right) d t \\
\vdots \\
u_{p 1}(x)=g_{p 1}(x)+\int_{\alpha_{p}}^{\beta_{p}} c_{p} k_{p}\left(x, t, u_{10}(t), u_{20}(t), \ldots, u_{p 0}(t)\right) d t
\end{array}\right.
$$

And also we take

$$
\left\{\begin{array}{c}
u_{1, s+1}(x)=\int_{\alpha_{1}}^{\beta_{1}} c_{1} k_{1}\left(x, t, u_{1, s}(t), u_{2, s}(t), \ldots, u_{p, s}(t)\right) d t \\
u_{2, s+1}(x)=\int_{\alpha_{2}}^{\beta_{2}} c_{2} k_{2}\left(x, t, u_{1, s}(t), u_{2, s}(t), \ldots, u_{p, s}(t)\right) d t \\
\vdots \\
u_{p, s+1}(x)=\int_{\alpha_{p}}^{\beta_{p}} c_{p} k_{p}\left(x, t, u_{1, s}(t), u_{2, s}(t), \ldots, u_{p, s}(t)\right) d t .
\end{array}\right.
$$

Or generally we have recursive relations as follows:

$$
\left\{\begin{array}{l}
u_{i 0}(x)=g_{i 0}(x), \\
u_{i 1}(x)=g_{i 1}(x)+\int_{\alpha_{i}}^{\beta_{i}} c_{i} k_{i}\left(x, t, u_{10}(t), u_{20}(t), \ldots, u_{p 0}(t)\right) d t \\
u_{i, s+1}(x)=\int_{\alpha_{i}}^{\beta_{i}} c_{i} k_{i}\left(x, t, u_{1, s}(t), u_{2, s}(t), \ldots, u_{p, s}(t)\right) d t \\
\quad i=1,2, \ldots, p s=1,2, \ldots
\end{array}\right.
$$




\section{NUMERICAL RESULTS}

In this study, we present two example for testing the accuracy of our proposed method for system of linear Fredholm integro-differential equation.

\section{Example 1:}

We consider the system

$$
\left\{\begin{array}{c}
u_{1}^{\prime \prime}(x)=\frac{3 x}{10}+6-\int_{0}^{1} 2 x t\left(u_{1}(t)-3 u_{2}(t)\right) d t \\
u_{2}^{\prime \prime}(x)=15 x+\frac{4}{5}-\int_{0}^{1} 3\left(2 x+t^{2}\right)\left(u_{1}(t)-2 u_{2}(t)\right) d t \\
u_{1}(0)=1, u_{2}(0)=-1, u_{1}^{\prime}(0)=0, u_{2}^{\prime}(0)=2
\end{array}\right.
$$

With the exact solution

$$
u_{1}(x)=3 x^{2}+1, u_{2}(x)=x^{3}+2 x-1 .
$$

Using inverse operation, we have

$$
\left\{\begin{array}{l}
u_{1}(x)=1+\frac{1}{20} x^{3}+3 x^{2}-\frac{1}{3} x^{3} \int_{0}^{1} t\left(u_{1}(t)-3 u_{2}(t)\right) d t \\
u_{2}(x)=-1+2 x+\frac{5}{2} x^{3}+\frac{2}{5} x^{2}-x^{3} \int_{0}^{1}\left(u_{1}(t)-2 u_{2}(t)\right) d t-\frac{3}{2} x^{2} \int_{0}^{1} t^{2}\left(u_{1}(t)-2 u_{2}(t)\right) d t .
\end{array}\right.
$$

Where $g_{1}(x)=1+3 x^{2}+\frac{1}{20} x^{3}, g_{2}(x)=-1+2 x+\frac{2}{5} x^{2}+\frac{5}{2} x^{3}$,

Now splitting $g_{1}(x)$ into two parts i.e., $g_{10}(x)=3 x^{2}+1, g_{11}(x)=\frac{1}{20} x^{3}$ and also splitting $g_{2}(x)$ into two parts i.e., $g_{20}(x)=x^{3}+2 x-1$ and $g_{21}(x)=\frac{3}{2} x^{3}+\frac{2}{5} x^{2}$ and using the recursive relations, we attain

$$
\left\{\begin{array}{l}
u_{10}(x)=3 x^{2}+1 \\
u_{20}(x)=x^{3}+2 x-1
\end{array}\right.
$$

And,

$$
\left\{\begin{array}{c}
u_{11}(x)=\frac{1}{20} x^{3}-\frac{1}{3} x^{3} \int_{0}^{1} t\left(u_{10}(t)-3 u_{20}(t)\right) d t=\frac{1}{20} x^{3}-\frac{1}{3} x^{3}\left(\frac{3}{20}\right)=0, \\
u_{21}(x)=\frac{3}{2} x^{3}+\frac{2}{5} x^{2}-x^{3} \int_{0}^{1}\left(u_{10}(t)-2 u_{20}(t)\right) d t-\frac{3}{2} x^{2} \int_{0}^{1} t^{2}\left(u_{10}(t)-2 u_{20}(t)\right) d t \\
\frac{3}{2} x^{3}+\frac{2}{5} x^{2}-\frac{3}{2} x^{3}-\frac{3}{2} x^{2}\left(\frac{4}{15}\right)=0,
\end{array}\right.
$$

And also we take

$$
\left\{\begin{array}{l}
u_{1, n+1}(x)=0, \\
u_{2, n+1}(x)=0,
\end{array} \quad n \geq 1\right.
$$

Thus using these into the series form (10),we get the solution under the following closed form

$$
u_{1}(x)=3 x^{2}+1, u_{2}(x)=x^{3}+2 x-1
$$

Which is the same as the exact solution. 


\section{Example 2:}

Suppose we want to solve the following system

$$
\left\{\begin{array}{c}
u_{1}^{\prime}(x)=-\frac{1}{4} x^{2}+\int_{0}^{1}\left[t e^{x} u_{1}(t)+t x^{2} u_{2}(t)\right] d t \\
u_{2}^{\prime}(x)=2 x+x \sin t-x \sin t e-\frac{1}{4} \cos x+\int_{0}^{1}\left[x \sin t u_{1}(t)+t \cos x u_{2}(t)\right] d t \\
u_{1}(0)=1, u_{2}(0)=0,
\end{array}\right.
$$

with the exact solution $u_{1}(x)=e^{x}, u_{2}(x)=x^{2}$.

By using inverse operation, we have

$$
\left\{\begin{array}{r}
u_{1}(x)=1-\frac{1}{12} x^{3}+\left(e^{x}-1\right) \int_{0}^{1} t u_{1}(t) d t+\frac{1}{3} x^{3} \int_{0}^{1} t u_{2}(t) d t \\
u_{2}(x)=x^{2}+\frac{1}{2} x^{2} \sin t-\frac{1}{2} x^{2} \sin t e-\frac{1}{4} \sin x+\frac{1}{2} x^{2} \int_{0}^{1} \sin t u_{1}(t) d t+\sin x \int_{0}^{1} t u_{2}(t) d t .
\end{array}\right.
$$

Where $g_{1}(x)=1-\frac{1}{12} x^{3}, g_{2}(x)=x^{2}+\frac{1}{2} x^{2} \sin t-\frac{1}{2} x^{2} \sin t e-\frac{1}{4} \sin x$,

now splitting $g_{1}(x)$ into two parts i.e., $g_{10}(x)=e^{x}, g_{11}(x)=1-\frac{1}{12} x^{3}-e^{x}$ and similarly splitting $g_{2}(x)$ into two parts i.e., $g_{20}(x)=x^{2}, g_{21}(x)=\frac{1}{2} x^{2} \sin t-\frac{1}{2} x^{2} \sin t e-\frac{1}{4} \sin x$ and by using the recursive relations, we get

$$
\left\{\begin{array}{l}
u_{10}(x)=g_{10}(x)=e^{x} \\
u_{20}(x)=g_{20}(x)=x^{2}
\end{array}\right.
$$

And,

$$
\left\{\begin{array}{l}
u_{11}(x)=1-\frac{1}{12} x^{3}-e^{x}+\left(e^{x}-1\right) \int_{0}^{1} t u_{10}(t) d t+\frac{1}{3} x^{3} \int_{0}^{1} t u_{20}(t) d t \\
=1-\frac{1}{12} x^{3}-e^{x}+\left(e^{x}-1\right)(1)+\frac{1}{3} x^{3}\left(\frac{1}{4} x^{3}\right)=0, \\
u_{21}(x)=\frac{1}{2} x^{2} \sin t-\frac{1}{2} x^{2} \sin t e-\frac{1}{4} \sin x+\frac{1}{2} x^{2} \int_{0}^{1} \sin t u_{10}(t)+\sin x \int_{0}^{1} t u_{20}(t) d t \\
=\frac{1}{2} x^{2} \sin t-\frac{1}{2} x^{2} \sin t e-\frac{1}{4} \sin x+\frac{1}{2} x^{2}(-\sin t+\sin t e)+\sin x\left(\frac{1}{4}\right)=0,
\end{array}\right.
$$

And also we take

$$
\left\{\begin{array}{l}
u_{1, n+1}(x)=0, \\
u_{2, n+1}(x)=0,
\end{array} \quad n \geq 1 .\right.
$$

Writing these into the series form (10), we have

$$
u_{1}(x)=e^{x}, u_{2}(x)=x^{2}
$$

which is the same as the exact solution. 


\section{CONCLUSION}

This paper presents the use of modified decomposition method for solving system of linear Fredholm Integro-differential equation. Exact solutions of the two test problems arising in many physical and biological models are calculated by using modified decomposition technique. We demonstrated that the modified decomposition procedure is quite efficient to determine the solution in closed form also.

\section{REFERENCES}

[1]. G. Adomian, Solving Frontier problem of Physics: The Decomposition Method, Kluwer Academic press, (1994).

[2]. A. Arikoglu and I. Ozkol, Solutions of Integral and Integro-Differential Equation Systems by Using Differential Transform Method, Computers Mathematics with Applications, Vol. 56, No. 9, 2411-2417, (2008).

[3]. T. Badredine, K. Abbaoui, Y. Cherruault, Convergence of Adomian's method applied to integral equations , Kybernetes, 28(5), (1999), 557-564.

[4]. J. Biazar, Solution of systems of integro-differential equation by Adomian decomposition method , Appl. Math. Comput. 168(2003), 1232-1238.

[5]. J. Biazar, H. Ghazvini, M. Eslami, Hes Homotopy perturbation method for systems of integrodifferential Equations, Chaos, Solitions and Fractals, Vol. 39, No. 3, (2009). 1253-1258.

[6]. Y. Cherruault, convergence of Adomian method ,kybernetes, Volume18(1989), 31-38.

[7]. S. Hyder Ali Muttaqi Shah, S.H. Sandilo, Modified Decompositin method for nonlinear VolterraFredholm integro-differential equation, Journal of Basic and Applied Sciences, Vol. 6, No. 1, 13-16, (2010).

[8]. K. Maleknejad, F. Mirzaee, S. Abbasbandy, Solving linear Integro-differential equations system by using rationalized Haar functions method, Applied Mathematics \& Computation, (2003).

[9]. K. Maleknejad and M. Tavassoli Kajani, Solving Linear integro-differential equation system by Galerkin methods with Hybrid functions, Applied Mathematics and Computation, Vol. 159, No. 3, (2004), 603-612.

[10]. J. Pour-Mahmoud, M.Y. Rahimi-Ardabili, S. Shahmorad, Numerical solution of the system of Fredholm integro-differential equations by the Tau method, Applied Mathematics and Computation, Vol. 168, No. 3,(2005), 465-478.

[11]. AM. Wazwaz, A reliable modification of Adomian's decomposition method, Appl Math Comput, (1999).

[12]. AM. Wazwaz, A reliable treatment for mixed Volterra-Fredholm integral equations, Appl Math Comput, (2002).

[13]. AM. Wazwaz, A first course In integral equations, World Scientific, Singapore, (1997). 